\section{Letter: Thrombotic Neurovascular Disease in COVID-19 Patients}

\section{To the Editor:}

Although the respiratory system is the primary target of the coronavirus, studies have demonstrated a strong tropism to the central nervous system (CNS). ${ }^{1,2}$ The severe acute respiratory syndrome coronavirus 2 (SARS-CoV-2) infects cells by binding to the angiotensin-converting enzyme 2 (ACE2) receptor. This receptor is also found in the CNS and plays a crucial role in autoregulating cerebral perfusion pressure. ${ }^{3,4}$ Additionally, epidemiological data demonstrated increased mortality due to cardiovascular and cerebrovascular diseases during flu pandemics due to a hypercoagulable state. ${ }^{5,6}$ The triad of neuroinvasion of SARS-CoV-2, induction of hypercoagulable state, ${ }^{5-9}$ and the inhibition of ACE2 blocking the formation of Angiotensin (17) serve as the pathophysiology for neurovascular insults. ${ }^{3,4} \mathrm{We}$ present a case series of coronavirus disease 2019 (COVID-19) patients from 2 health systems developing cerebrovascular insult.

\section{METHODS}

\section{Study Design}

Institutional Review Board (IRB) approved the study protocol and waived the need for informed consent. A retrospective analysis was conducted across 2 institutions between March 20 and April 10, 2020. Fourteen patients were identified with the diagnosis of cerebrovascular insult and COVID-19 infection. COVID-19 was diagnosed using reverse-transcriptase/polymerase-chain-reaction assays of nasopharyngeal samples for SARS-CoV-2. Medical charts were queried for baseline patient characteristics, comorbidities, COVID-19 symptoms, duration between COVID-19 symptoms and the neurological manifestation, cerebrovascular insult, National Institutes of Health Stroke Scale (NIHSS) at presentation, procedure details, and mortality.

\section{Data Sharing Statement}

The relevant anonymized patient-level data are available on reasonable request from the authors.

\section{Ethical Approval}

All procedures performed in the studies involving human participants were per the ethical standards of the IRB or National Research Committee, and the 1964 Helsinki Declaration and its later amendments or comparable ethical standards.

\section{Informed Consent}

The study protocol was reviewed and approved by the Thomas Jefferson University Institutional Review Board. Following our institutional guidelines, all protected health information were removed, and individual patient consent was not required for the analysis of this case series.

\section{RESULTS}

The total sample size was 14 patients. The mean age was $60.1 \pm 11.1 \mathrm{yr}$, and 9 patients were males (64.3\%). Six patients (42.8\%) had no significant prior medical history. Seven patients $(50.3 \%)$ had neurological insult as the initial manifestation of COVID-19. The average duration between the onset of COVID19 symptoms and the cerebrovascular insult was $3.5 \mathrm{~d}$ (range: $0-17)$. The cerebrovascular pathologies were 12 cases of acute ischemic stroke (AIS) and 2 cases of sinus thrombosis. The mean NIHSS was 15.8 (range: 1-30), and all patients were treated within $6 \mathrm{~h}$ of symptoms onset. A total of 4 patients had carotid T occlusions, 2 had tandem occlusion (internal carotid artery [ICA] and middle cerebral artery [MCA] M1 occlusion), 1 patient had M1 and A2 occlusion, 2 patients had M1 occlusion, 2 patients had M2 occlusion, 2 patients had sinus thrombosis, and 1 patient had central retinal artery occlusion. Two patients developed hemorrhagic conversion requiring decompressive surgery. The mean duration of the mechanical thrombectomy (MT) procedures was 95.5 min (range: 17-428), and a favorable thrombolysis in cerebral infarction (TICI) score $(>2 \mathrm{~b})$ was achieved in all patients. The total mortality incidence was $6(42.8 \%$; Table).

\section{DISCUSSION}

Despite the small sample size, the data demonstrate some unusual trends worth sharing, at least to draw awareness. First, the mean age of the population was $60 \mathrm{yr}, 42 \%$ of the cohort was younger than 55 , and $42 \%$ did not have any traditional cerebrovascular risk factors. Moreover, cerebrovascular insult was the primary manifestation of COVID-19 in patients who did not have the fulminant virus manifestations present. Occlusions occurred in multiple arterial territories and both on the arterial and on the venous sides; the latter are more challenging to treat, and the procedures require additional expertise. Selected procedures were more complex and challenging, requiring multiple attempts to retrieve the clot entirely due to the severe clot burden. Such an observation is evident in the relatively long procedure time compared to historical data. Also, mortality rates reached $42.8 \%$, with TICI $\geq 2 \mathrm{~b}$ achieved in all cases. Two patients developed hemorrhagic conversion requiring decompressive hemicraniectomy (Figure 1).

The level of causality between the SARS-CoV-2 and AIS is yet to be determined. However, maintaining a vigilant attitude is crucial, specifically if it leads to favorable outcomes. It is early to determine the exact impact of COVID-19 on the incidence of acute cerebrovascular diseases. The SARS-CoV-2 is neuroinvasive and neurovirulent (Figure 2), $, 1,2$ binds to ACE2 and reduces its downstream effect (Figure 3),, 3 and induces a hyperinflammatory response characterized by a cytokine storm 


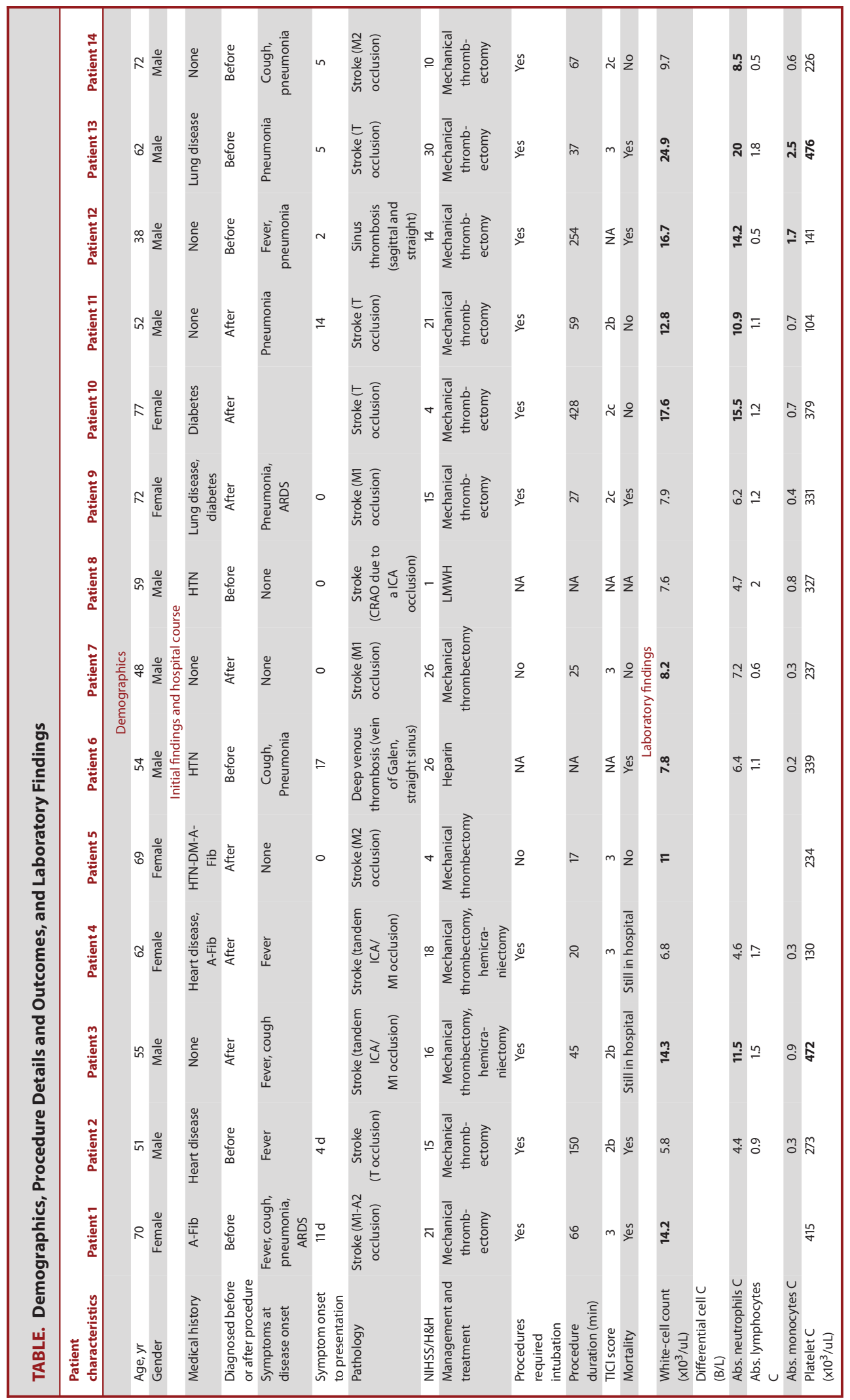








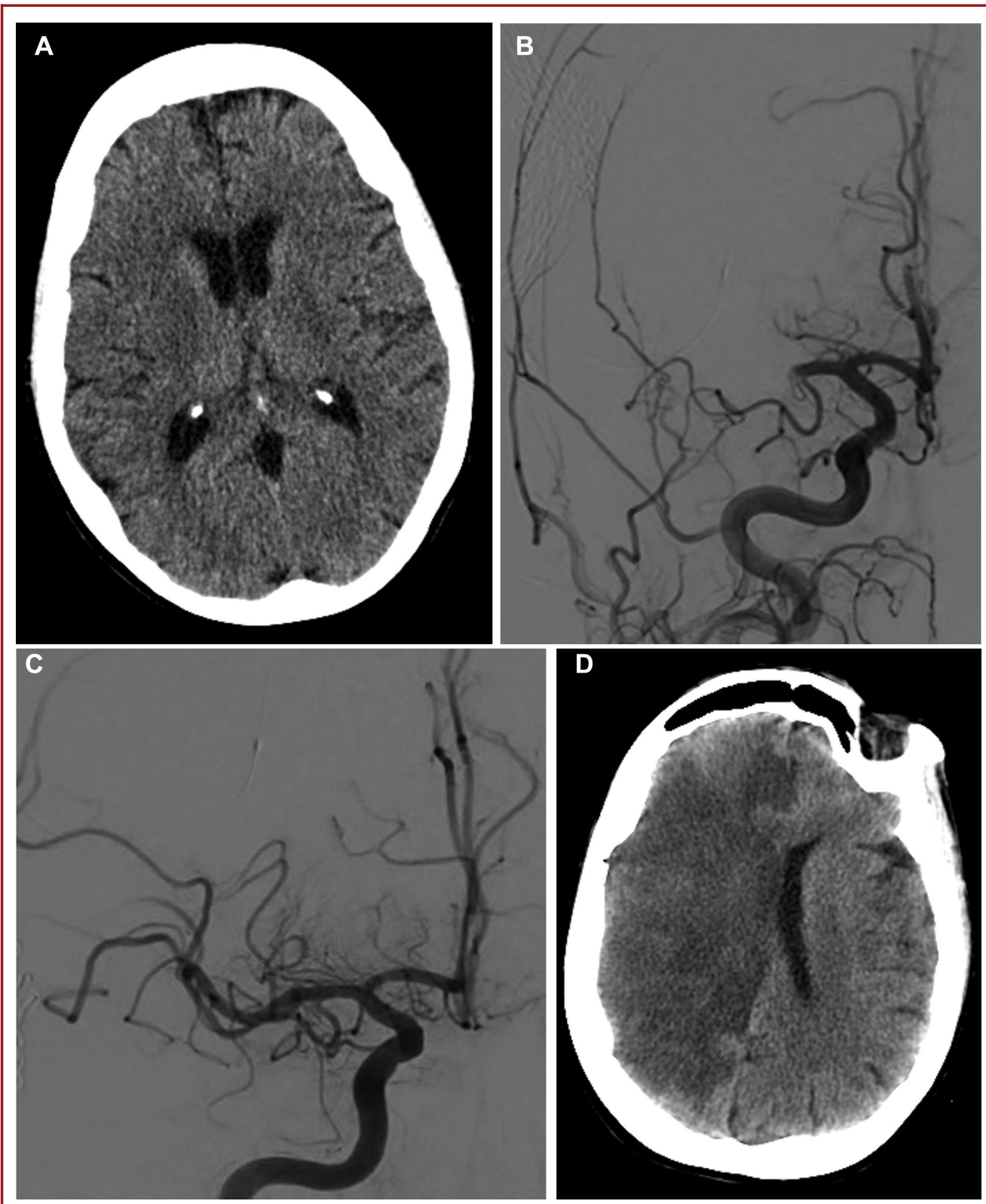

FIGURE 1. A patient in the seventh decade with a history of A-Fib on eliqus was being treated for COVID-19, and on the 11 th day of treatment, the patient developed an acute neurological insult. Tissue plasminogen activator was not given because of anticoagulation, and head computed tomography (CT) did not show hemorrhage. The patient was transferred and underwent a mechanical thrombectomy procedure within $4 \mathrm{~h}$ and $18 \mathrm{~min}$. The insult progressed into complete infarct, and the patient passed away 3 d later. A, Noncontrast axial view of brain CT scan showing no hemorrhage. B, Anteroposterior (AP) digital subtraction angiography (DCA) of a right ICA injection showing an $M 1$ and an A2 occlusion. $\mathrm{C}, A P D C A$ showing complete revascularization of both vessels (TICI 3). D, Noncontrast axial view of the brain day 1 post mechanical thrombectomy showing the progression of the insult into a complete infarct. 

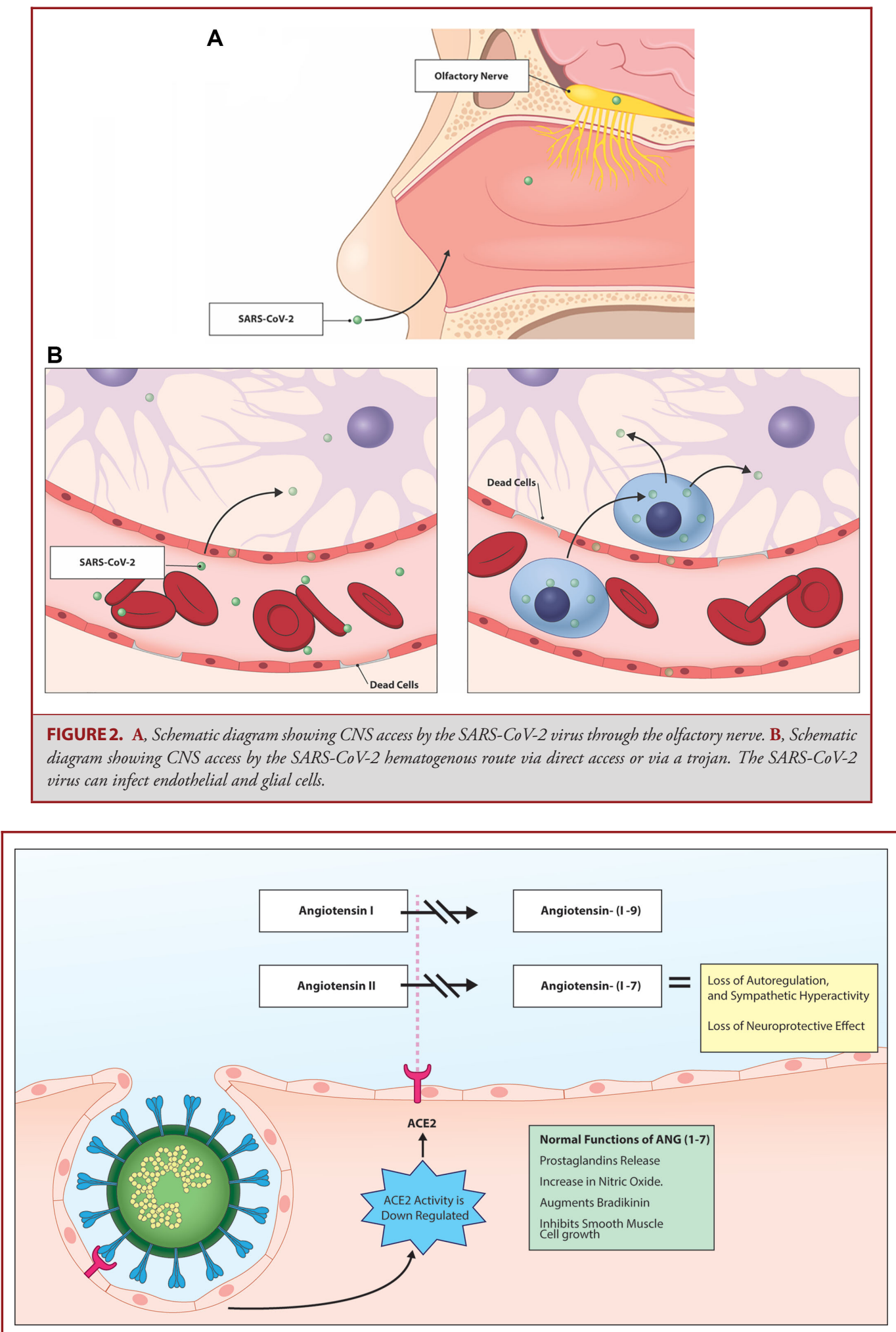

FIGURE 3. Schematic diagram at the level of CNS endothelium showing the SARS-CoV-2 induced ACE2 receptor downregulation. This inhibits the conversion of angiotensin I and II into their active metabolites, Angiotensin (1-9) and Angiotensin (1-7), respectively. The decline in Angiotensin (1-7) levels leads to loss of neuroprotective effects and sympathetic hyperactivity. 


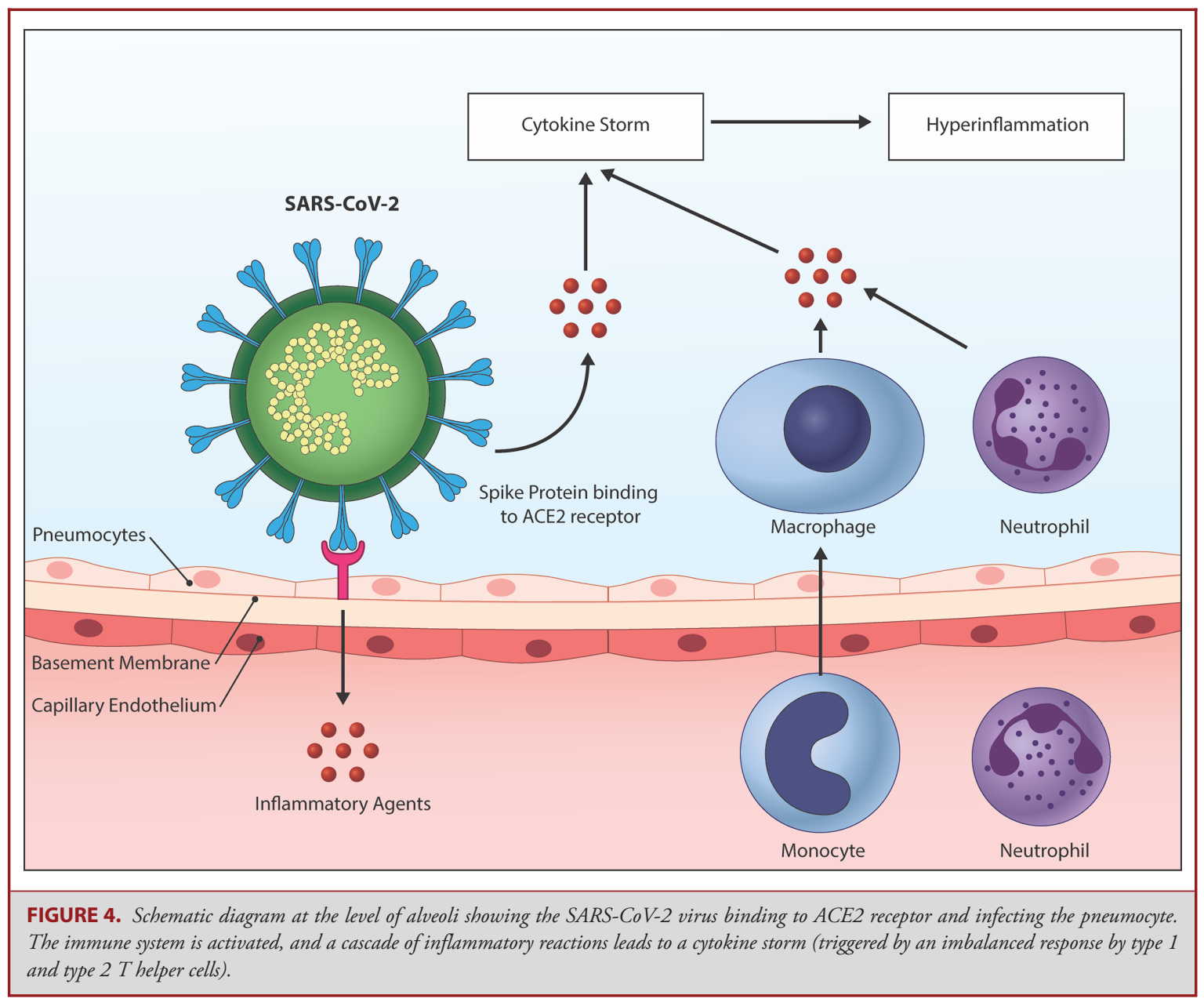

(Figure 4). ${ }^{7-9}$ This leads to vasculitis, ${ }^{10}$ increased sympathetic tone, arrhythmias, and a hypercoagulable state.

\section{CONCLUSION}

Unusual trends have been seen in AIS patients who are COVID-19 positive; while it is too early to establish direct causality, our preliminary data can be used to raise awareness in the population. We are seeing younger patients with no risk factors presenting with AIS and MT procedures are more challenging to deal with. It is crucial in pandemic times to watch and follow closely noteworthy trends and investigate and report new findings.

\section{Disclosures}

Dr Jabbour is a consultant for Medtronic and MicroVention. Dr Tjoumakaris and Dr Gooch are consultants for Stryker. The other authors have no personal, financial, or institutional interest in any of the drugs, materials, or devices described in this article.

Ahmad Sweid, MD (i)* $^{*}$

Batoul Hammoud, $\mathrm{MD}^{\ddagger}$ Joshua H. Weinberg, BS* Mazen Oneissi, MD $^{\S \Phi}$ Eytan Raz, MD\|

Maksim Shapiro, $\mathrm{MD}^{\| \#}$

Maureen DePrince, BA* $^{*}$ Stavropoula Tjoumakaris, MD ${ }^{*}$ Michael R. Gooch, MD* Nabeel A. Herial, MD, MPH* Hekmat Zarzour, MD ${ }^{*} *$ Victor Romo, MD**

Robert H. Rosenwasser, MD* Pascal Jabbour, MD $\oplus^{*}$ * Department of Neurological Surgery Thomas Jefferson University Hospital Philadelphia, Pennsylvania

${ }^{\ddagger}$ Department of Pediatric Endocrinology Children Hospital of Philadelphia Philadelphia, Pennsylvania 


\section{${ }^{S}$ Department of Neurosurgery Baylor College of Medicine \\ Houston, Texas \\ Jan and Dan Duncan Neurological Research Institute \\ Texas Children's Hospital \\ Houston, Texas \\ "Department of Radiology \\ New York University Langone Medical Center \\ New York, New York \\ \#Department of Neurosurgery \\ New York University Langone Medical Center \\ New York, New York \\ ** Department of Anesthesia \\ Thomas Jefferson University Hospital \\ Philadelphia, Pennsylvania}

\section{REFERENCES}

1. Lau K-K, Yu W-C, Chu C-M, Lau S-T, Sheng B, Yuen K-Y. Possible central nervous system infection by SARS coronavirus. Emerg Infect Dis. 2004;10(2):342.

2. Bergmann CC, Lane TE, Stohlman SA. Coronavirus infection of the central nervous system: host-virus stand-off. Nat Rev Microbiol. 2006;4(2):121-132.
3. Zhang H, Penninger JM, Li Y, Zhong N, Slutsky AS. Angiotensin-converting enzyme 2 (ACE2) as a SARS-CoV-2 receptor: molecular mechanisms and potential therapeutic target. Intensive Care Med. 2020;46(4):586-590.

4. Xu P, Sriramula S, Lazartigues E. ACE2/ANG-(1-7)/Mas pathway in the brain: the axis of good. Am J Physiol Regul Integr Comp Physiol. 2011;300(4):R804-R817.

5. Urbanek C, Palm F, Grau AJ. Influenza and stroke risk: a key target not to be missed? Infect Disord Drug Targets. 2010;10(2):122-131.

6. Toschke AM, Heuschmann PU, Wood O, Wolfe CD. Temporal relationship between influenza infections and subsequent first-ever stroke incidence. Age Ageing. 2009;38(1):100-103.

7. Mehta P, McAuley DF, Brown M, Sanchez E, Tattersall RS, Manson JJ. COVID19: consider cytokine storm syndromes and immunosuppression. Lancet North Am Ed. 2020;395(10229):1033-1034.

8. Connors JM, Levy JH. Thromboinflammation and the hypercoagulability of COVID-19. J Thromb Haemost. published online: April 17, 2020. (doi:10.1111/jth.14849)

9. Zhang Y, Xiao M, Zhang S, et al. Coagulopathy and antiphospholipid antibodies in patients with Covid-19. N Engl J Med. 2020;382(17):e38.

10. Varga Z, Flammer AJ, Steiger $P$, et al. Endothelial cell infection and endotheliitis in COVID-19. Lancet North Am Ed. 2020;395(10234):14171418.

Copyright (c) 2020 by the Congress of Neurological Surgeons

$10.1093 /$ neuros/nyaa254 